\title{
More Zeros of Certain Bessel Functions of Fractional Order
}

The following table is a continuation of the one given by M. ABraMowitz

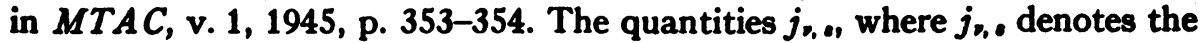
sth zero of $J_{v}(x)$, are given below for $v= \pm \frac{1}{1}, \pm \frac{1}{y_{1}} \pm \frac{z}{z_{1}} \pm \frac{i}{k}$, and for $s=[8(1) 30 ; 10 D]$. These values are believed to be correct to well within a unit in the tenth decimal. Thus this table supplements the one given by

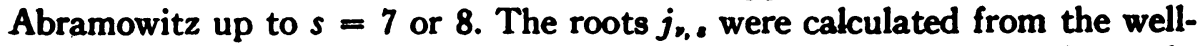
known asymptotic expansion (see $M T A C$ p. 354), carrying $12 \mathrm{D}$ in the work.

An interesting check was performed upon these roots by obtaining the first difference as a function of $s$ and then obtaining the divided differences of those first differences as functions of $\nu$. Apart from the first entry corresponding to $s=8$ and which was checked independently, the divided differences (which were, in turn, also differenced as a function of $s$ where they were largest, i.e. for $s$ near 8), showed an error very much within 2000 units in the twelfth decimal place. Since the error in the roots was multiplied by a probable factor of $\mathbf{2 0 0}$ or more as a result of the process of taking the divided difference, the roots in the original manuscript are probably correct to about a unit in the 11 th decimal place.

Most of the calculations on these roots were performed by Miss RUTH Zucker and Mrs. Ruth Capuano.

\begin{tabular}{|c|c|c|c|c|}
\hline \multicolumn{5}{|c|}{ Tables of $j_{n, 0}$} \\
\hline s & $D=1$ & $v=-t$ & $p=1$ & $x=-1$ \\
\hline $\begin{array}{r}8 \\
9 \\
10 \\
11 \\
12\end{array}$ & $\begin{array}{l}24.7438277961 \\
27.8849946034 \\
31.0262474761 \\
34.1675627296 \\
37.3089246363\end{array}$ & $\begin{array}{l}23.9585534953 \\
27.0996936674 \\
30.2409276522 \\
33.3822290291 \\
36.5235804430\end{array}$ & $\begin{array}{l}24.8737314228 \\
28.0150117149 \\
31.1563548787 \\
34.2977436760 \\
37.4391666407\end{array}$ & $\begin{array}{l}23.8266562471 \\
26.9679102166 \\
30.1092347166 \\
33.2506098032 \\
36.3920224013\end{array}$ \\
\hline $\begin{array}{l}13 \\
14 \\
15 \\
16 \\
17\end{array}$ & $\begin{array}{l}40.4503223437 \\
43.5917481224 \\
46.7331963179 \\
49.8746626986 \\
53.0161440346\end{array}$ & $\begin{array}{l}39.6649700250 \\
42.8063893836 \\
45.9478324186 \\
49.0892945893 \\
52.2307724460\end{array}$ & $\begin{array}{l}40.5806158496 \\
43.7220856531 \\
46.8635719141 \\
50.0050715339 \\
53.1465821455\end{array}$ & $\begin{array}{l}39.5334635829 \\
42.6749270440 \\
45.8164082072 \\
48.9579036683 \\
52.0994108430\end{array}$ \\
\hline $\begin{array}{l}18 \\
19 \\
20 \\
21 \\
22\end{array}$ & $\begin{array}{l}56.1576378181 \\
59.2991420721 \\
62.4406552173 \\
65.5821759767 \\
68.7237033066\end{array}$ & $\begin{array}{l}55.3722633210 \\
58.5137651192 \\
61.6552761718 \\
64.7967951336 \\
67.9383209080\end{array}$ & $\begin{array}{l}56.2881019100 \\
59.4296293768 \\
62.5711633865 \\
65.7127030012 \\
68.8542474541\end{array}$ & 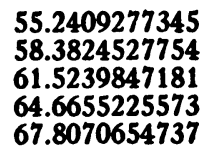 \\
\hline $\begin{array}{l}23 \\
24 \\
25 \\
26 \\
27\end{array}$ & $\begin{array}{l}71.8652363457 \\
75.0067743771 \\
78.1483167988 \\
81.2898631020 \\
84.4314128537\end{array}$ & $\begin{array}{l}71.0798525921 \\
74.2213894359 \\
77.3629308110 \\
80.5044761872 \\
83.6460251137\end{array}$ & $\begin{array}{l}71.9957961121 \\
75.1373484480 \\
78.2789040192 \\
81.4204624512 \\
84.5620234254\end{array}$ & $\begin{array}{l}70.9486127933 \\
74.0901639563 \\
77.2317184937 \\
80.3732760100 \\
83.5148361692\end{array}$ \\
\hline $\begin{array}{l}28 \\
29 \\
30\end{array}$ & $\begin{array}{l}87.5729656827 \\
90.7145212696 \\
93.8560793373\end{array}$ & $\begin{array}{l}86.7875772052 \\
89.9291321301 \\
93.0706896015\end{array}$ & $\begin{array}{l}87.7035866686 \\
90.8451519455 \\
93.9867190522\end{array}$ & $\begin{array}{l}86.6563986839 \\
89.7979633070 \\
92.9395298248\end{array}$ \\
\hline
\end{tabular}


Tables of $j_{r, 0}-$ Contivued

\begin{tabular}{rc}
\multicolumn{1}{r}{$s$} & $=\boldsymbol{I}$ \\
8 & 25.3907144312 \\
9 & 28.5327278949 \\
10 & 31.6746579988 \\
11 & 34.8165272733 \\
12 & 37.9583508024 \\
& \\
13 & 41.1001390646 \\
14 & 44.2418995658 \\
15 & 47.3836378232 \\
16 & 50.5253579832 \\
17 & 53.6670632220 \\
& \\
18 & 56.8087560137 \\
19 & 59.9504383142 \\
20 & 63.0921116899 \\
21 & 66.2337774103 \\
22 & 69.3754365150 \\
23 & 72.5170898635 \\
24 & 75.6587381727 \\
25 & 78.8003820451 \\
26 & 81.9420219908 \\
27 & 85.0836584448 \\
28 & 88.2252917800 \\
29 & 91.3669223180 \\
30 & 94.5085503377
\end{tabular}

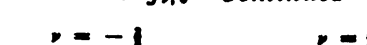

23.2959758671

26.4380633449

29.5800458826

32.7219536520

35.8638062730

39.0056170547

42.1473953433

45.2891478947

$\mathbf{4 8 . 4 3 0 8 7 9 7 1 3 6}$

$\mathbf{5 1 . 5 7 2 5 9 4 5 8 6 2}$

$\mathbf{5 4 . 7 1 4 2 9 5 4 2 9 8}$

57.8559845286

60.9976636963

64.1393343918

67.2809978012

70.4226548994

73.5643064946

76.7059532629

$\mathbf{7 9 . 8 4 7 5 9 5 7 7 3 7}$

82.9892345106

86.1308698863

89.2725022556

92.4141319250
25.5193219366

28.6615845511

31.8037149655

34.9457487864

38.0877098894

41.2296148817

44.3714756721

47.5133010223

50.6550975224

$\mathbf{5 3 . 7 9 6 8 7 0 2 2 3 9}$

56.9386230642

60.0803591576

63.2220809997

66.3637906137

$\mathbf{6 9 . 5 0 5 4 8 9 6 5 7 2}$

72.6471795012

75.7888612893

78.9305359832

82.0722043975

85.2138672265

88.3555250659

91.4971784295

$\mathbf{9 4 . 6 3 8 8 2 7 7 6 3 0}$
. $=-1$

23.1625059341

26.3049025693

29.4471278984

32.5892313867

35.7312451311

38.8731908670

42.0150838362

45.1569350434

48.2987526323

$\mathbf{5 1 . 4 4 0 5 4 2 7 5 8 6}$

$\mathbf{5 4 . 5 8 2 3 1 0 1 6 2 0}$

$\mathbf{5 7 . 7 2 4 0 5 8 5 5 0 8}$

60.8657908680

64.0075094793

67.1492163076

70.2909129325

73.4326006631

76.5742805938

79.7159536465

$\mathbf{8 2 . 8 5 7 6 2 0 6 0 3 6}$

85.9992821328

89.1409388079

92.2825911246

150 Nassau Street

NYMTP

\section{RECENT MATHEMATICAL TABLES}

299[A].-Mathematical Cuneiform Texts, edited by O. E. Neugebauer and A. J. SACrs. (American Oriental Series, volume 29.) New Haven, Conn., Amer. Oriental So., 329 Sterling Memorial Library, and the Amer. Schools of Oriental Research, $1945 . x, 177$ p. +49 plates. $\$ 5.00$

In Quellen u. Studien zur Geschichte der Mathematik . . ., A. Quellen, v. 3, (MKT I-III) 1935-37, Otto Neugebauer, the outstanding authority on ancient mathematics and astronomy, published a monumental pioneer work on Mathematical Cuneiform Texts, tablets in collections of Berlin, Brussels, Istanbul, Jena, Leyden, London, New Haven, Oxford, Paris, Philadelphia, Strassburg, and Toronto. Nearly $\mathbf{4 0 0 0}$ years ago the Babylonians knew how to find the positive roots of quadratic equations; how to solve simultaneous linear and quadratic equations; and how to calculate compound interest; and they knew that the angle in a semi-circle is a right angle, and our familiar relation between the hypotenuse and sides of a right-angled triangle.

The present work in English is a detailed study of about 200 tablets in the United States and nearly a dozen in Europe. New material of great interest is presented.

In all Babylonian mathematical and astronomical work, tables were fundamental, especially tables for multiplication, squares, square roots, cubes and cube roots, and tables of reciprocals. Since 60 was basic in the sexagesimal notation of the Babylonians, if $n$ were a number written in this notation, $n=1 / n$ could be expressed in a finite number of terms (e.g. $\overline{9}=0 ; 6,40$ ), or in a never ending series of terms (e.g. $\overline{7}=0 ; 8,34,17,8,34,17 \ldots$ with $8,34,17$ being repeated indefinitely). The necessary and sufficient condition for the first case is that $n$ must be of the form $n=2^{\alpha} 3^{\beta} 5^{\gamma}$, where $\alpha, \beta, \gamma$ are integers or zero. Such numbers are called regular numbers. In a Louvre tablet dating from about 350 B.C. there are 